\title{
ASYMPTOTIC BEHAVIOR OF OSCILLATING RADIAL SOLUTIONS TO CERTAIN NONLINEAR EQUATIONS*
}

\author{
CHANGFENG GUI ${ }^{\dagger}$ AND FENG ZHOU ${ }^{\ddagger}$
}

Key words. Bessel function, Allen-Cahn equations and thin-film equations, oscillation.

AMS subject classifications. 35B45, 35J60, 35B05

1. Introduction. Recently, in the investigation of thin film problems, a class of oscillating radial solutions has attracted the attention. The radial solutions of concern satisfy the following initial value problem:

$$
u^{\prime \prime}+\frac{n-1}{r} u^{\prime}=f(u) \quad \text { in } \mathbb{R}_{+}, \quad u(0)=\alpha>0, \quad u^{\prime}(0)=0 .
$$

where $f \in C^{1}(0, \infty)$ satisfies the following general conditions:

(i) $f$ has a single zero $t_{0}$ in $(0, \infty)$ satisfying $f^{\prime}\left(t_{0}\right)<0$,

(ii) $f$ is nonincreasing near 0 and $\lim _{t \rightarrow 0^{+}} f(t)=\infty$.

The radial solutions is a special case of more general thin film problem in a bounded domain $\Omega$ in $\mathbb{R}^{n}$ with Neumann boundary condition

$$
\Delta u=f(u), \quad x \in \Omega, \quad \frac{\partial u}{\partial \nu}=0 \text { on } \partial \Omega .
$$

A typical example is that $f(u)=u^{-p}-\mu_{1} u^{-q}-\mu_{2}$ with constants $p>\max (q, 0)$. This kind of semilinear equation appears in several applications in mechanics and physics. In particular, it has been used to model the dynamics of thin films for viscous liquids. Some detailed physics background can be found in [1]-[3], [9] and [12]-[14]. Some recent mathematical analysis can be found in $[4,6,7,8,10,11,15,17]$ and the references therein.

It was proved in [8] (see also [11]) that in dimension $N \geq 3$, for each $\alpha \in\left(0, t_{0}\right)$, (1.1) has a unique positive solution $u_{\alpha}$. Moreover, $u_{\alpha}$ oscillates around the constant $t_{0}$, that is, there is an increasing positive sequence $\left\{r_{\alpha}^{n}\right\}$ such that $\{r \in(0, \infty)$ : $\left.u_{\alpha}^{\prime}(r)=0\right\}=\left\{r_{\alpha}^{n}\right\}$, and $\lim _{n \rightarrow \infty} r_{\alpha}^{n}=\infty$. Here $r_{\alpha}^{2 i+1}$ are local maxima of $u_{\alpha}$ with $u_{\alpha}\left(r_{\alpha}^{2 i+1}\right)>t_{0}$ for any $i \in \mathbb{N}$; while $r_{\alpha}^{2 i}$ are local minima with $u_{\alpha}\left(r_{\alpha}^{i}\right)<t_{0}$. It is also proven that there exists a singular (or so-called a rupture) radial solution $u_{0}(r)$ of (1.1) such that $u_{0} \in C\left(\mathbb{R}^{N}\right), u_{0}(0)=0, u_{0}(r)>0$ for $r \in(0, \infty)$ and $f\left(u_{0}\right) \in L_{l o c}^{1}\left(\mathbb{R}^{N}\right)$. Moreover, any singular radial solution of (1.1) is oscillatory around $t_{0}$ and converges to $t_{0}$ as $r \rightarrow \infty$.

In [11], Jiang and Ni have showed the existence and uniqueness of radial rupture solution for $f(u)=u^{-p}-\mu_{2}$ in $\mathbb{R}^{N}$ with $p, \mu_{2}>0$ and $N \geq 2$. Moreover, they proved that $\lim _{n \rightarrow \infty} r_{\alpha}^{n+1}-r_{\alpha}^{n}$ exits, and hence obtained the asymptotic formula for the length of oscillating interval, which depends only on $p$ and $\mu_{2}$.

\footnotetext{
*Received June 10, 2008; accepted for publication October 21, 2008.

† School of Mathematics, Hunan University, Changsha, PRC and Department of Mathematics, U-9, University of Connecticut, Storrs, CT 06269, USA (gui@math.uconn.edu).

¥Department of Mathematics, East China Normal University, Shanghai, China (fzhou@math. ecnu.edu.cn).
} 
It it a natural question that whether more accurate asymptotic behaviors of the radial solutions can be obtained. Similar question also arises in the study of oscillating radial solutions of Allen Cahn equation

$$
\Delta u+u-u^{3}=0, \quad x \in \mathbb{R}^{n} .
$$

Generally speaking, it is important to understand radial entire solutions since they display one of the fundamental structures near interesting points such as singularities of solutions. The asymptotic behavior of an entire solution at infinity is an essential property since it reveals the condition away from the singularity. There has been a lot of literature on the asymptotic behavior of positive radial solutions, see, for example [16], [5], [18], etc. However, it seems that little has been done on oscillating radial solutions.

In this note, we shall show an accurate asymptotic behavior of radial solutions for a vast class of equations in term of Bessel functions. In Section 1, some basic information on Bessel functions are collected; In Section 2, we show, for small initial data, the existence of oscillating radial solutions which behaves like Bessel functions at infinity; In Section 3 and 4, we shall show that the asymptotic behavior of radial solutions in term of Bessel functions for rather general equations including Allen-Cahn equations and thin-film equations.

\section{Preliminaries.}

2.1. Bessel Function. We recall that the Bessel Function

$$
x^{2} y^{\prime \prime}+x y^{\prime}+\left(x^{2}-\alpha^{2}\right) y=0 \quad x>0 .
$$

It has two independent (linearly) solutions: Bessel function of the first kind and second kind, $J_{\alpha}(x), Y_{\alpha}(x)$ with asymptotic expansions

$$
\begin{aligned}
J_{\alpha}(x) \sim \sqrt{\frac{2}{\pi x}}[ & \cos \left(x-\frac{\alpha \pi}{2}-\frac{\pi}{4}\right) \sum_{j=0}^{\infty} \frac{(-1)^{j}(\alpha, 2 j)}{(2 x)^{2 j}} \\
& \left.-\sin \left(x-\frac{\alpha \pi}{2}-\frac{\pi}{4}\right) \sum_{j=0}^{\infty} \frac{(-1)^{j}(\alpha, 2 j+1)}{(2 x)^{2 j+1}}\right]
\end{aligned}
$$

and

$$
\begin{aligned}
Y_{\alpha}(x) \sim \sqrt{\frac{2}{\pi x}}[ & \sin \left(x-\frac{\alpha \pi}{2}-\frac{\pi}{4}\right) \sum_{j=0}^{\infty} \frac{(-1)^{j}(\alpha, 2 j)}{(2 x)^{2 j}} \\
& \left.+\cos \left(x-\frac{\alpha \pi}{2}-\frac{\pi}{4}\right) \sum_{j=0}^{\infty} \frac{(-1)^{j}(\alpha, 2 j+1)}{(2 x)^{2 j+1}}\right]
\end{aligned}
$$

where

$$
(\alpha, k)= \begin{cases}\frac{\left(4 \alpha^{2}-1\right)\left(4 \alpha^{2}-3^{2}\right) \cdots\left(4 \alpha^{2}-(2 k-1)^{2}\right)}{2^{k} k !}, & k>1 \\ 1, & k=0 .\end{cases}
$$

Moreover, the Wronskian determinant associated is

$$
W\left(J_{\alpha}(x), Y_{\alpha}(x)\right)=\frac{2}{\pi x} .
$$


2.2. Radial solution for a linear equation. Now consider the linear Laplace equation

$$
\Delta u+\beta^{2} u=0, \quad x \in \mathbb{R}^{n},
$$

its radial solution $u(r)$ satisfies

$$
u^{\prime \prime}+\frac{n-1}{r} u^{\prime}+\beta^{2} u=0 \quad r=|x|>0 .
$$

Let $u=r^{\frac{2-n}{2}} y(\beta r)$, then $y(x)$ satisfies (2.1) with $\alpha=\frac{n-2}{2}$. Hence (2.7) has two linearly independent solutions

$$
u_{1}(r)=r^{\frac{2-n}{2}} J_{\alpha}(\beta r), \quad u_{2}(r)=r^{\frac{2-n}{2}} Y_{\alpha}(\beta r)
$$

and the Wronskian determinant is

$$
W\left(u_{1}(r), u_{2}(r)\right)=u_{1} u_{2}^{\prime}-u_{2} u_{1}^{\prime}=\frac{2}{\pi} r^{1-n} .
$$

In particular, we have

$$
\begin{aligned}
& u_{1}(r)=\frac{2}{\sqrt{\pi \beta}} r^{\frac{1-n}{2}}\left[\cos \left(\beta r-\frac{n-1}{4} \pi\right)+O\left(\frac{1}{r}\right)\right] \\
& u_{2}(r)=\frac{2}{\sqrt{\pi \beta}} r^{\frac{1-n}{2}}\left[\sin \left(\beta r-\frac{n-1}{4} \pi\right)+O\left(\frac{1}{r}\right)\right] .
\end{aligned}
$$

3. Existence of Radial Solutions for Nonlinear Equation. Let us consider the nonlinear problems

$$
\begin{cases}u^{\prime \prime}+\frac{n-1}{r} u^{\prime}+\beta^{2} u+f(u)=0, & r>0 \\ u(r) \rightarrow 0, & \text { as } r \rightarrow \infty\end{cases}
$$

where $f(0)=f^{\prime}(0)=0$.

We suppose that $f(u) \in C^{1, \sigma}\left(-\delta_{0}, \delta_{0}\right)$, for some $\delta_{0}>0, \sigma>\frac{2}{n-1}$ if $n>3$, and $f(u) \in C^{2, \sigma}\left(-\delta_{0}, \delta_{0}\right)$, if $n=3, \sigma>0$.

It is easy to see that (3.1) is equivalent to

$$
\begin{aligned}
u(r)= & u_{1}(r) \int_{R}^{r} \frac{\pi}{2} s^{n-1} u_{2}(s)(-f(u(s))) \mathrm{d} s \\
& -u_{2}(r) \int_{R}^{r} \frac{\pi}{2} s^{n-1} u_{1}(s)(-f(u(s))) \mathrm{d} s+\gamma_{1} u_{1}(r)+\gamma_{2} u_{2}(r),
\end{aligned}
$$

or we can write

$$
u(r)=\frac{\pi}{2} \int_{R}^{r} s^{n-1} f(u(s))\left[u_{2}(r) u_{1}(s)-u_{1}(r) u_{2}(s)\right] \mathrm{d} s+\gamma_{1} u_{1}(r)+\gamma_{2} u_{2}(r) \quad r \geq R .
$$

Then we have the following 
THEOREM 3.1. When $\left|\left(\gamma_{1}, \gamma_{2}\right)\right|$ is small enough, (3.3) has a unique solution $u_{\gamma_{1}, \gamma_{2}}$ such that

$$
u_{\gamma_{1}, \gamma_{2}}=\left(\tilde{\gamma}_{1}+o(1)\right) u_{1}+\left(\tilde{\gamma}_{2}+o(1)\right) u_{2}
$$

for some $\left(\tilde{\gamma}_{1}, \tilde{\gamma}_{2}\right)$, provided that one of the following conditions holds

$$
\begin{aligned}
& \left\{\begin{array}{rlrl}
\text { a) }: f(u) \in C^{1, \sigma}\left(-\delta_{0}, \delta_{0}\right), & f(0)=f^{\prime}(0)=0, & \sigma>\frac{2}{n-1} \text { if } n>3 \text { or } \\
\text { b) }: f(u) \in C^{2, \sigma}\left(-\delta_{0}, \delta_{0}\right), f(0)=f^{\prime}(0)=f^{\prime \prime}(0)=0, & \sigma>0 \text { if } n=3 \text { or }
\end{array}\right. \\
& \text { c) : } f(u) \in C^{3, \sigma}\left(-\delta_{0}, \delta_{0}\right), f(0)=f^{\prime}(0)=f^{\prime \prime}(0)=f^{(3)}(0)=0, \sigma>0 \text { if } n=2 \text {. }
\end{aligned}
$$

Proof. We define a Banach space $H_{R}$ for any $R>0$.

$$
H_{R}=\left\{u \in C(R, \infty) ; \sup _{r \geq R}\left|u(r) r^{\frac{n-1}{2}}\right|<\infty\right\}
$$

with a weighted norm

$$
\|u\|_{H_{R}}=\sup _{r \geq R}\left|u(r) r^{\frac{n-1}{2}}\right| .
$$

It is easy to see that \|\|$_{H_{R}}$ is a norm in $H_{R}$. Define now an operator in $H_{R}$ for any $\left(\gamma_{1}, \gamma_{2}\right)$ by

$$
K_{\gamma_{1}, \gamma_{2}}(u)(r)=\frac{\pi}{2} \int_{R}^{r} s^{n-1} f(u(s))\left[u_{2}(r) u_{1}(s)-u_{1}(r) u_{2}(s)\right] \mathrm{d} s+\gamma_{1} u_{1}(r)+\gamma_{2} u_{2}(r) .
$$

Then there exists $\sigma_{0}, \delta_{0}$ such that if $\left|\gamma_{1}\right|+\left|\gamma_{2}\right|<\sigma_{0}, \delta<\delta_{0}$, there holds

$$
\left\|K_{\gamma_{1}, \gamma_{2}}(u)\right\|_{H_{R}} \leq \delta \quad \text { when }\|u\|_{H_{R}}<\delta .
$$

In fact,

$$
\begin{aligned}
\left\|K_{\gamma_{1}, \gamma_{2}}(u)\right\|_{H_{R}} & \leq \frac{4}{\pi \beta} \frac{\pi}{2} \sup _{r \geq R} \int_{R}^{r} s^{n-1}|f(u(s))| s^{-\frac{n-1}{2}} \mathrm{~d} s+2 \sqrt{\frac{2}{\pi \beta}}\left(\left|\gamma_{1}\right|+\left|\gamma_{2}\right|\right) \\
& \leq 2 \sqrt{\frac{2}{\pi \beta}}\left(\left|\gamma_{1}\right|+\left|\gamma_{2}\right|\right)+\frac{4}{\beta} \sup _{r \geq R} \int_{R}^{r}\left|s^{\frac{n-1}{2}} u(s)\right|\left|\frac{f(u(s))}{u(s)}\right| \mathrm{d} s \\
& \leq 2 \sqrt{\frac{2}{\pi \beta}}\left(\left|\gamma_{1}\right|+\left|\gamma_{2}\right|\right)+\frac{4 M}{\beta} \sup _{r \geq R} \int_{R}^{r}\left|s^{\frac{n-1}{2}} u(s)\right||u(s)|^{\alpha} \mathrm{d} s \\
& \leq 2 \sqrt{\frac{2}{\pi \beta}}\left(\left|\gamma_{1}\right|+\left|\gamma_{2}\right|\right)+\frac{4 M}{\beta}\|u\|_{H_{R}}^{1+\alpha}\left(\sup _{r \geq R} \int_{R}^{r} s^{-\frac{n-1}{2} \alpha} \mathrm{d} s\right) \\
& \leq 2 \sqrt{\frac{2}{\pi \beta}}\left(\left|\gamma_{1}\right|+\left|\gamma_{2}\right|\right)+\frac{C}{\beta}\|u\|_{H_{R}}^{1+\alpha} R^{1-\frac{n-1}{2} \alpha},
\end{aligned}
$$

where $\alpha=\sigma$ in case a) in (3.4); $\alpha=1+\sigma$ in case $\mathbf{b})$ in (3.4), and $\alpha=2+\sigma$ in case c) in (3.4) and

$$
C=4 M /\left(\frac{n-1}{2} \alpha-1\right) .
$$


Here we have used

$$
\begin{aligned}
& \left|\frac{f(u)}{u^{1+\alpha}}\right| \leq M, \\
& \left|\frac{f(u)-f(v)}{u-v}\right| \leq M \max \left\{|u|^{\alpha},|v|^{\alpha}\right\},
\end{aligned}
$$

for $u$ near $u=0$ and $\alpha>\frac{2}{n-1}$.

Therefore, when

$$
\begin{aligned}
& \left|\gamma_{1}\right|+\left|\gamma_{2}\right|<\gamma_{0}:=\sqrt{\frac{\pi \beta}{2}} \frac{1}{4} \delta \\
& \delta<\delta_{0}:=\left(\frac{\beta}{4 C}\right)^{\frac{1}{\alpha}} R^{-\frac{1}{\alpha}+\frac{n-1}{2}}
\end{aligned}
$$

we have

$$
\left\|K_{\gamma_{1}, \gamma_{2}}(u)\right\|_{H_{R}} \leq \frac{\delta}{2}+\delta^{1+\alpha} \times \frac{C}{\beta} R^{1-\frac{n-1}{2} \alpha} \leq \frac{\delta}{2}+\frac{\delta}{4}<\delta, \quad \text { when }\|u\|_{H_{R}}<\delta .
$$

Furthermore, we know $K_{\gamma_{1}, \gamma_{2}}$ is a contracting mapping in $B_{\delta}(0) \subset H_{R}$, since

$$
\begin{aligned}
\left\|K_{\gamma_{1}, \gamma_{2}}(u)-K_{\gamma_{1}, \gamma_{2}}(v)\right\|_{H_{R}} & \leq \frac{4}{\beta} \sup _{r \geq R} \int_{R}^{r} s^{\frac{n-1}{2}}|f(u(s))-f(v(s))| \mathrm{d} s \\
& \leq \frac{4 M}{\beta}\|u-v\|_{H_{R}} \delta^{\alpha}\left(\sup _{r \geq R} \int_{R}^{r} s^{-\frac{n-1}{2} \alpha} \mathrm{d} s\right) \\
& \leq \frac{1}{2}\|u-v\|_{H_{R}} .
\end{aligned}
$$

Here we have used (3.8).

It is easy to see that (3.8) and (3.11) hold if (3.4) is provided. Therefore $K_{\gamma_{1}, \gamma_{2}}(u)$ has a fixed point in $B_{\delta}(0) \subset H_{R}$ when $\left(\gamma_{1}, \gamma_{2}\right)$ and $\delta$ satisfies (3.9).

Furthermore, the integral in (3.3) is integrable with $r$ replaced by $\infty$. Hence Theorem 3.1 is proven with $\left(\tilde{\gamma}_{1}, \tilde{\gamma}_{2}\right)$ sufficiently small when $R$ is fixed. Note that if we allow $R$ to be large, the size of $\gamma_{1}, \gamma_{2}$ can be indeed large as long (3.9) holds. $\square$

4. Application to Allen-Cahn Equation. Consider the radial solution to Allen-Cahn equation

$$
\left\{\begin{array}{l}
u^{\prime \prime}+\frac{n-1}{r} u^{\prime}-F^{\prime}(u)=0, \quad r=|x|, x \in \mathbb{R}^{n} \\
u(0)=u_{0}, \quad\left|u_{0}\right|<1 .
\end{array}\right.
$$

where $F(u) \in C^{2, \sigma}([-1,1])$, and satisfies

$$
\left\{\begin{array}{l}
F^{\prime}(1)=F^{\prime}(-1)=0, \quad F(1)=F(-1)=0 \\
F(u)>0 \quad \text { if }|u|<1 \\
F^{\prime}(0)=0, F^{\prime \prime}(0)<0, F^{\prime}(u)<0 \quad \text { if } 0<u<1 \\
F^{\prime}(u)>0 \quad \text { if }-1<u<0 .
\end{array}\right.
$$


We have then

$$
\left[\left(\frac{u^{\prime}(r)}{2}\right)^{2}-F(u(r))\right]^{\prime}=-\frac{n-1}{r} u^{\prime 2} \leq 0, \quad \forall r>0,
$$

and thus

$$
F(u(r)) \geq F\left(u_{0}\right)+\left(\frac{u^{\prime}(r)}{2}\right)^{2}>0 \quad \forall r>0 .
$$

Hence

$$
|u(r)|<1, \quad \forall r>0 .
$$

LEMma 4.1. We have

$$
\lim _{r \rightarrow \infty} u(r)=0 .
$$

Proof. If not, we assume that there exists $r_{N} \rightarrow \infty$ such that

$$
u\left(r_{N}\right) \rightarrow \alpha \neq 0, \quad u^{\prime}\left(r_{N}\right) \rightarrow 0 \text { as } n \rightarrow \infty .
$$

Define

$$
u_{n}(r)=u\left(r+r_{N}\right) \quad r>0 .
$$

We know by the standard elliptic theory that

$$
|\nabla u| \leq C, \quad\left|\nabla^{2} u\right| \leq C, \quad\|u\|_{C^{2, \sigma}\left(\mathbb{R}^{2}\right)}<C .
$$

Then $u_{n}(r) \rightarrow u_{\infty}(r)$ in $C^{2}(0, \infty)$ up to a subsequence, and $u_{0}(r)$ satisfies $\left|u_{\infty}(r)\right|<$ $\left|F^{-1}\left(F\left(u_{0}\right)\right)\right|<1, \forall r \geq 0$, and

$$
\left\{\begin{array}{l}
u_{\infty}^{\prime \prime}(r)-F^{\prime}\left(u_{\infty}(r)\right)=0, \quad r>0 \\
u_{\infty}(0)=\alpha \neq 0, \quad u_{\infty}^{\prime}(0)=0, \quad|\alpha|<1
\end{array}\right.
$$

Equation (4.8) can be solved easily by integration, the only solution are periodic solutions.

$$
u_{\infty}(r)=P_{\alpha}(r) \quad \text { with }\left\{\begin{array}{l}
P_{\alpha}\left(r+T_{\alpha}\right)=P_{\alpha}(r), \text { for some } T_{\alpha}>0 \\
P_{\alpha}(0)=\alpha .
\end{array}\right.
$$

Without loss of generality, we assume $\alpha>0$. Let $r_{k}$ be the $k$-th local maximum point of $u(r)$ in $(0, \infty)$. Then $u\left(r_{k}\right)$ is decreasing in $k$, since

$$
F\left(u\left(r_{k+1}\right)\right) \geq F\left(u\left(r_{k}\right)\right), \quad u\left(r_{k}\right)>0 .
$$

Hence $\lim _{k \rightarrow \infty} u\left(r_{k}\right)=\alpha>0$. We can then prove

$$
\left\|u\left(r_{k+1}+r\right)-P_{\alpha}(r)\right\|_{C^{2, \alpha}\left(\left[0, T_{\alpha}\right]\right)} \rightarrow 0 \quad \text { as } k \rightarrow \infty .
$$


On the other hand, (4.3) leads to

$$
\begin{aligned}
F\left(u\left(r_{k}\right)\right)-F\left(u\left(r_{N}\right)\right) & =\int_{r_{N}}^{r_{k}} \frac{n-1}{r}\left|u^{\prime}(r)\right|^{2} \mathrm{~d} r \\
& =(n-1) \sum_{i=N}^{k-1} \int_{r_{i}}^{r_{i+1}} \frac{\left(u^{\prime}(r)\right)^{2}}{r} \mathrm{~d} r \\
& \geq \frac{n-1}{2} \int_{0}^{T_{\alpha}}\left|P_{\alpha}^{\prime}(r)\right|^{2} \mathrm{~d} r \sum_{i=N}^{k-1} \frac{1}{r_{i+1}},
\end{aligned}
$$

when $N$ is sufficiently large. Letting $k \rightarrow \infty$, we have a contradiction. Hence Lemma 4.1 is proven. $\square$

Let $m_{k}=u\left(r_{k}\right)$. We know that $m_{k}$ is decreasing in $k$ and tends to 0 as $k$ goes to infinity. Next we shall show

Lemma 4.2. For any fixed $\epsilon>0$, we have

$$
m_{k} \leq C k^{-\frac{n-1}{2}+\epsilon}
$$

Hence

$$
u(r) \leq C r^{-\frac{n-1}{2}+\epsilon}, \quad r>0
$$

Proof. Let $\beta^{2}=-F^{\prime \prime}(0)$. First we note $u\left(r_{k}+r\right) / m_{k}$ converges to $\cos (\beta r)$ uniformly in any bounded interval as $k$ tends to infinity. Indeed we have

$$
\left\|u(r)-m_{k} \cos \left(\beta\left(r-r_{k}\right)\right)\right\|_{C^{1}\left[r_{k} r_{k}+T_{0}\right]}=o(1) m_{k}
$$

where $T_{0}=\frac{2 \pi}{\beta}$ and

$$
r_{k+1}-r_{k}=T_{0}+o(1)
$$

Furthermore, we have

$$
F(0)-F\left(m_{k}\right)=\frac{1}{2}\left(\beta^{2}+o(1)\right) m_{k}^{2} .
$$

and

$$
\begin{aligned}
& \int_{r_{k}}^{r_{k+1}} \frac{n-1}{r}\left|u^{\prime}(r)\right|^{2} \mathrm{~d} r \\
& =\int_{0}^{T_{0}} \frac{n-1}{k\left(T_{0}+o(1)\right)} m_{k}^{2} \beta^{2} \sin ^{2}(\beta x) d x \\
& =\left(\frac{n-1}{2}+o(1)\right) \beta^{2} \frac{m_{k}^{2}}{k} .
\end{aligned}
$$

Since

$$
F(0)-F\left(m_{k}\right)=\int_{r_{k}}^{\infty} \frac{n-1}{r}\left|u^{\prime}(r)\right|^{2} \mathrm{~d} r .
$$


Then, for any fixed small constant $\epsilon$ and $k$ large enough we have

$$
m_{k}^{2} \geq(n-1-2 \epsilon) \sum_{i=k}^{\infty} \frac{m_{i}^{2}}{i} .
$$

This leads to (4.13) by the following calculus lemma. $\mathrm{Q}$

LEMma 4.3. Let $\left\{a_{k}\right\}$ be sequence of nonincreasing positive numbers and satisfies

$$
a_{k} \geq p \sum_{i=k}^{\infty} \frac{a_{i}}{i}
$$

for some positive constant $p$. Then for some positive constant $C$ there holds

$$
a_{k} \leq C k^{-p} .
$$

Proof. Define

$$
A_{k}=\sum_{i=k}^{\infty} \frac{a_{i}}{i}, \quad k \geq 1 .
$$

Then

$$
k\left(A_{k}-A_{k+1}\right) \geq p A_{k}, \quad k \geq 1 .
$$

Hence

$$
\frac{A_{k}}{A_{k+1}} \geq \frac{k}{k-p}, \quad k>p .
$$

Let $N_{0}>p$ fixed. For any integer $N>N_{0}$, we have

$$
\frac{A_{N_{0}}}{A_{N}} \geq \prod_{k=N_{0}}^{k=N-1} \frac{k}{k-p} \geq \prod_{k=N_{0}}^{k=N-1}\left(1+\frac{p}{k}\right) .
$$

Then

$$
\ln \left(A_{N-0}\right)-\ln \left(A_{N}\right) \geq \sum_{k=N_{0}}^{N-1} \ln \left(1+\frac{p}{k}\right) \geq p \ln (N)-c, \quad N>N_{0}
$$

where $c$ is a constant independent of $N$. Therefore we obtain

$$
A_{N} \leq C N^{-p}, \quad \forall N>0
$$

where $C$ is a constant independent of $N$. Since $a_{i}$ is nonincreasing, we obtain

$$
A_{k}-A_{2 k+1}=\sum_{i=k}^{2 k} \frac{a_{i}}{i} \geq \frac{1}{2} a_{2 k}, \quad \forall k .
$$

Hence the lemma is proven. $\square$

Now we apply Theorem 3.1 to obtain 
Theorem 4.4. Assume $f(u)=F^{\prime \prime}(0) u-F^{\prime}(u)$ satisfies (3.4). Then when $\left|u_{0}\right|<1$, the solution $u(r)$ to (4.1) has asymptotic behavior

$$
u(r)=\left(\tilde{\gamma}_{1}+o(1)\right) u_{1}(r)+\left(\tilde{\gamma}_{2}+o(1)\right) u_{2}(r)
$$

where $u_{1}, u_{2}$ are defined in (2.10).

Proof. Just note that we choose $R$ large enough. Define $\gamma_{1}, \gamma_{2}$ by the following relation

$$
\left\{\begin{array}{l}
\gamma_{1} u_{1}(R)+\gamma_{2} u_{2}(R)=u(R) \\
\gamma_{1} u_{1}^{\prime}(R)+\gamma_{2} u_{2}^{\prime}(R)=u^{\prime}(R)
\end{array}\right.
$$

Following Lemma 4.2 with $\epsilon<\frac{n-1}{2}-\frac{1}{\alpha}$, we see that $\left(\gamma_{1}, \gamma_{2}\right)$ satisfies (3.9) when $R$ is large enough. Hence $(3.2)$ with $\left(\gamma_{1}, \gamma_{2}\right)$ has a solution $\bar{u}(r)$ with asymptotic behavior (4.18). On the other hand, by the uniqueness of initial value problem for (4.1), we have $u(r)=\bar{u}(r)$, the theorem is proved.

REMARK 4.5. In the typical Allen-Cahn equation, we have $f(u)=-u^{3}$, which satisfies (3.4) when $n \geq 3$. However, when $n=2$ the condition (3.4) is not satisfied. It would be interesting to show that (4.18) still holds in this case. Similarly, if we consider $f(u)=|u|^{p-1} u$ with $p>1$, it would be interesting to show that (4.18) still holds for $n \geq 2$. Note that when $n=1$, the solution may not vanish, hence (4.18) does not hold in general.

5. Application to the thin film equation. We return to the study of the thin film equations. Consider

$$
\left\{\begin{array}{l}
u^{\prime \prime}+\frac{n-1}{r} u^{\prime}=g(u) \quad r>0 \\
u(0)=u_{0}>0, \quad u^{\prime}(0)=0 .
\end{array}\right.
$$

where the nonlinear term $g(u)$ satisfies

$$
g^{\prime}(1)<0, \quad g(1)=0, g(u)>0 \text { for } 0<u<1, \quad g(u)<0 \text { for } u>1 .
$$

Let $G(u)=\int_{1}^{u} g(s) \mathrm{d} s$, then $G(u)$ is non increasing for $u>1$ and non decreasing for $u<1$. Note that $g(u)=u^{-p}-u^{-q}$ with $p>q \geq 0$ is a typical example.

It is easy to see that

Lemma 5.1. $u$ is bounded above and below away from 0 .

Proof. Note that

$$
\left(\frac{u^{\prime 2}}{2}-G(u)\right)^{\prime}=-\frac{n-1}{r}\left(u^{\prime}\right)^{2} \leq 0, \quad r>0 .
$$

Then

$$
G(u(r))>G\left(u_{0}\right)+\frac{u^{\prime}(r)^{2}}{2} \geq G\left(u_{0}\right), \quad r \geq 0 .
$$

By (5.2), we know $0<\delta<u(r)<C<\infty, \forall r$ for some $\delta, C>0$.

We can also obtain the limit of $u$ easily. 
LEMma 5.2. There holds

$$
\lim _{r \rightarrow \infty} u(r)=1
$$

Proof. Consider $v(r)=u(r)-1$, then it satisfies

$$
v^{\prime \prime}+\frac{n-1}{r} v^{\prime}-g^{\prime}(1) v+f(v)=0 \quad r>0,
$$

where

$$
f(v)=-g(1+v)+g^{\prime}(1) v, \quad-g^{\prime}(1)=\beta^{2}>0 .
$$

Then the arguments in Lemma 4.1 leads to (5.5).

TheOREm 5.3. Assume (5.2) and (3.4) where $f(v)$ is given by (5.7). Then the solution $u$ of (5.1) has the following asymptotic behavior

$$
u(r)=1+\left(\tilde{\gamma}_{1}+o(1)\right) u_{1}(r)+\left(\tilde{\gamma}_{2}+o(1)\right) u_{2}(r), \quad \text { as } r \rightarrow \infty .
$$

Proof. The proof is similar to proof of Theorem 4.4. The detail is omit. $\square$

REMARK 5.4. In the typical thin film equation, we have $g(u)=u^{-p}-1$ and $f(v)=-(1+v)^{-p}+1-p v$, which satisfies (3.4) when $n>3$. However, when $n=2,3$ the condition (3.4) is not satisfied. It would be interesting to investigate if (4.18) still hold in this case.

Acknowledgements. The first author is partially supported by NSF Grant DMS 0500871. The research is also supported in part by NSFC (No. 10528103), NNSF (No. 10671071) of China and the "basic research project (973, No. 2006CB805902) of China".

Note added at galley proof. Similar results for the cases $n=2,3$ as in Remark 4.5 and Remark 5.4 have been proven by Changfeng Gui, Xue Luo and Feng Zhou in a forthcoming paper.

\section{REFERENCES}

[1] A.L. Bertozzi And M.C. Pugh, Long-wave instabilities and saturation in thin film equations, Comm. Pure Appl. Math., 51 (1998), pp. 625-661.

[2] A.L. Bertozzi And M.C. Pugh, Finite-time blow-up of solutions of some long-wave unstable thin film equations, Indiana Univ. Math. J., 49 (2000), pp. 1323-1366.

[3] J.P. Burelbach, S.G. Bankoff and S.H. Davis, Nonlinear stability of evaporating /condensing liquid films, J. Fluid Mech., 195 (1988), pp. 463-494.

[4] G. GRün, Droplet spreading under weak slippage-existence for the Cauchy problem, Comm. Partial Diff. Equa., 29 (2004), pp. 1697-1744.

[5] C. Gui, W.M. Ni And X.F. Wang, On the stability and instability of positive steady states of a semilinear heat equation in $\mathbb{R}^{N}$, Comm. Pure Appl. Math., 45 (1992), pp. 1153-1181.

[6] H.X. Guo, Z.M. Guo And K. LI, Positive solutions of a semilinear elliptic equation with singular nonlinearity, J. Math. Anal. Appl., 323 (2006), pp. 344-359.

[7] Z.M. Guo AND J.C. WeI, Symmetry of nonnegative solutions of a semilinear elliptic equation with singular nonlinearity, Proc. Royal. Soc. Edinburgh. Section A, 137 (2007), pp. 963994.

[8] Z.M. Guo, D. Ye AND F. ZHou, Existence of singular positive solutions for some semilinear elliptic equations, Pacific J. Math., 236 (2008), pp. 57-71. 
[9] C.C. Hwang, C.K. Lin And W.Y. Uen, A nonlinear three-dimensional rupture theory of thin liquid films, J. Colloid Interf. Sci., 190 (1997), pp. 250-252.

[10] H.Q. JiAng AND F.H. Lin, Zero set of Sobolev functions with negative power of integrability, Chinese Ann. Math. Ser. B, 25 (2004), pp. 65-72.

[11] H.Q. JIANG AND W.M. NI, On steady states of van der Waals force driven thin film equations, European J. Applied Math., 18 (2007), pp. 1-28.

[12] R.S. Laugesen and M.C. Pugh, Properties of steady states for thin film equations, Europ. J. Appl. Math., 11 (2000), pp. 293-351.

[13] R.S. LAugesen And M.C. Pugh, Finite-time blow-up of solutions of some long-wave unstable thin film equations, Indiana Univ. Math. J., 49 (2000), pp. 1323-1366.

[14] R.S. Laugesen AND M.C. Pugh, Linear stability of steady states for thin film and CahnHilliard type equations, Arch. Rat. Mech. Anal., 154 (2000), pp. 3-51.

[15] K. Li, H.X. Guo And Z.M. Guo, Positive single rupture solutions to a semilinear elliptic equation, Appl. Math. Letters, 18 (2005), pp. 1177-1183.

[16] Y. LI, Asymptotic behavior of positive solutions of equation $\Delta u+K(x) u^{p}=0$ in $\mathbb{R}^{N}$, J. Diff. Equa., 95 (1992), pp. 304-330.

[17] D. SlepČEv AND M.C. Pugh, Selfsimilar blowup of unstable thin-film equations, Indiana Univ. Math. J., 54 (2005), pp. 1697-1738.

[18] X. WANG, On the Cauchy problem for reaction-diffusion equations, Trans. Amer. Math. Soc., 337 (1993), pp. 549-589. 
C. GUI AND F. ZHOU 